\title{
Online Appendix Gender Homophily in Referral Networks: Consequences for the Medicare Physician Earnings Gap
}

\author{
By Dan Zeltzer*
}

\section{A. Mathematical Appendix \\ 1. Defining Relative Homophily with Weighted Links}

Relative homophily can be easily adapted to accommodate weighted links. First, define $n_{g G}$ using weighted degrees, as follows: Let $n_{j k}$ be the weight of the link from $j$ to $k$ (e.g. number of patients referred). The weighted out-degree of $j$ is $d(j)=\sum_{k} n_{j k}$. The weighted out-degree to females is $d^{F}(j)=\sum_{k} \mathbb{1}_{g_{k}=F} n_{j k}$. Now $n_{m F}$ is the average of $d^{F} / d$ over all male $j$, and so on for $n_{g G}$. The rest of the definition is as previously indicated in Section III.A.

\section{The Importance of Link Direction in Defining Homophily}

Considering a directed network as if it is undirected is undesirable when studying homophily. Figure A1 illustrates the sensitivity of relative homophily to link direction.

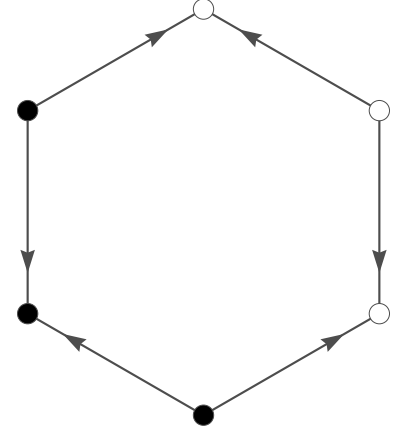

(a) Relative Homophily

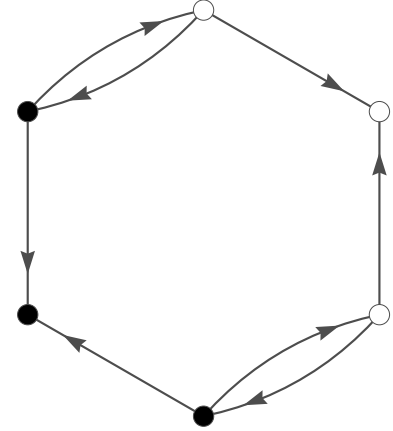

(b) No Relative Homophily

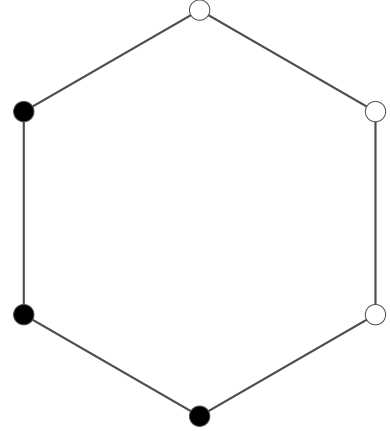

(c) Common

Undirected Representation

Figure A1. Homophily and Link Direction

Notes: Because referrals are asymmetric, link direction is important for defining relative homophily: the network (a) exhibits relative homophily while (b) does not, a difference concealed in their undirected counterpart (c). More generally, this example speaks against treating asymmetric relationships as if they were symmetric when studying homophily.

Source: Author's calculations

Neglecting link directions may also give rise to spurious inbreeding homophily. Consider the directed network of referrals among $2 N$ physicians, exactly half of which are doctors. Denote as

* Tel Aviv University, School of Economics, Tel Aviv, Israel 6997801 (dzeltzer@tauex.tau.ac.il). 
before by $m$ and $M$ the fractions of male doctors and male specialists. Assume no gender bias: all doctors make the same average number of total referrals and send a fraction $M$ of their referrals to male specialists. Suppose that we ignore directions, and consider the undirected version of this network. Formally, let the degree of each node in the undirected representation be the sum of out-degree and in-degree in the directed networks. In such case, although there is no gender bias in referrals, the undirected representation would exhibit inbreeding homophily, unless the gender mix of doctors and specialists happens to be equal. To see this, without loss of generality normalize the overall number of referrals (links) to one. Assuming referrals are gender-neutral, the gender composition of referrals is simply a function of the physician population gender shares (Table A1). In contrast to the directed version of the referral network (Case B), in the undirected version (Case A) there is no distinction between male-to-female and female-to-male referrals, of which there are in total $m(1-M)+(1-m) M$. Hence, in the undirected network there are only three possible gender compositions of physicians involved in a referral: both male, both female, and mixed gender. inbreeding homophily is zero when the fraction of ingroup referrals by men equals their population fraction. In this example, the population fraction of male physicians is $(m+M) / 2$. Therefore, inbreeding homophily is zero when

$$
\frac{m M}{m M+\frac{1}{2}(m(1-M)+(1-m) M)}=\frac{m+M}{2}
$$

which holds if and only if $m=M$. In all other cases, inbreeding homophily is nonzero, despite the fact that, by construction, referrals are unbiased.

Table A1-Directed and Undirected Representations of Unbiased Referrals

\begin{tabular}{|c|c|c|}
\hline \multicolumn{2}{|c|}{ Referral Gender Composition } & \multirow[b]{2}{*}{ Fraction of Referrals } \\
\hline A. Undirected Network & B. Directed Network & \\
\hline both male & male to male & $m M$ \\
\hline & male to female & $m(1-M)$ \\
\hline mixed gender \{ & female to male & $(1-m) M$ \\
\hline both female & female to female & $(1-m)(1-M)$ \\
\hline
\end{tabular}

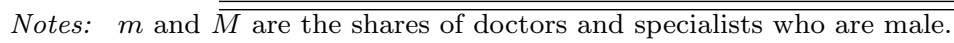

Source: Author's calculations

\section{Interpreting The Common Tendency to Refer to Male Specialists as Bias}

The analysis in Section III.C maintained that the tendency common to both genders to refer more to males reflects unobserved heterogeneity in specialist characteristics that makes male specialists more appropriate referral targets. This section considers the other extreme interpretation, and considers all such common tendencies to refer to men as a bias in favor of men (and against women) that is common to both genders. Table A7 shows that under this assumption, at the current estimated gender-specific bias $\beta$ and gender shares, the gender bias in referrals contributes 20.1 percentage points to the physician gender earnings gap.

Formally, Table A7 shows estimates obtained from rederiving (10) with a common term $e^{\delta \mathbb{1}_{g_{k}}=m}$, viz., including the estimated $\hat{\delta}=0.165$ in the bias. Linearly approximating the gap around $\beta \approx$ 
$0, \delta \approx 0$ provides some intuition about the first-order effect of a common bias $\delta$ :

$$
G a p \approx \delta+\left(1-\left(M-\frac{1}{2}\right) 2 \delta\right)\left(m-\frac{1}{2}\right) 2 \beta+O\left(\beta^{2}\right)+O\left(\delta^{2}\right)
$$

With $M=0.5$, (1) boils down to $\delta+\kappa$, where $\kappa=\left(m-\frac{1}{2}\right) 2 \beta$ is the first-order gap as it appears in (10). With $M=1$, it boils down to $\delta+(1-\delta) \kappa$. Namely, for the estimated levels of bias, the first-order effect of a common bias is approximately an additive shift in demand toward male and away from female specialists. Table A7 does not rely on this approximation and shows the exact calculations, which include small nonlinear effects.

\section{Proofs}

Proof of Proposition 1.

\section{PROOF:}

First consider the homogeneous case: $\delta=0$, and note that the conditional probabilities of referrals to a male specialist are:

$$
P\left(M_{m}\right)=\frac{M}{M+\omega(1-M)} \geq M \geq \frac{\omega M}{\omega M+(1-M)}=P\left(M_{f}\right)
$$

where $P\left(G_{g}\right):=P\left(g_{k}=G \mid g_{j}=g\right)$ denotes the probability that the chosen specialist's gender is $G$ conditional on doctors' gender being $g$, and $\omega=e^{-\beta} \in(0,1]$.

Where $P\left(M_{m}\right)$ and $P\left(M_{f}\right)$ are derived by summing up probabilities of referrals to all available specialists. E.g., for $g_{j}=m$ :

$$
\begin{aligned}
P\left(M_{m}\right) & =\sum_{k: g_{k}=M} P\left(Y_{j k}=1\right)=\frac{\sum_{k: g_{k}=M} e^{\beta \mathbb{1}_{g_{j}=g_{k}}}}{\sum_{k} e^{\beta \mathbb{1}_{g_{j}}=g_{k}}} \\
& =\frac{\sum_{k: g_{k}=M} e^{\beta}}{\sum_{k: g_{k}=M} e^{\beta}+\sum_{k: g_{k} \neq M} e^{0}}=\frac{M e^{\beta}}{M e^{\beta}+1-M} .
\end{aligned}
$$

For all $M \in(0,1)$, biased preferences result in relative homophily, $P\left(M_{m}\right)>P\left(M_{f}\right)$ : doctors of each gender slightly discount the other (by a factor $\omega) .{ }^{1}$ Conversely, with unbiased preferences $(\beta=0)$, relative homophily is zero, as $P\left(M_{m}\right)=M=P\left(M_{f}\right)$.

Consider next the case: $\delta \neq 0$, where a correlation exists between gender and decision-relevant specialist characteristics (e.g., men may be more experienced or women may be available for fewer hours). In this case, (1) becomes:

$$
P\left(M_{m}\right)=\frac{M}{M+\omega \eta(1-M)} \geq \frac{\omega M}{\omega M+\eta(1-M)}=P\left(M_{f}\right)
$$

\footnotetext{
${ }^{1}$ Clearly, if specialists are mostly men then men refer more to men than to women: $P\left(M_{m}\right)>P\left(F_{m}\right)$, which is not to be confused with $P\left(M_{m}\right)>P\left(M_{f}\right)$.
} 
which holds, because:

$$
\begin{aligned}
P\left(M_{m}\right) & =\frac{\sum_{k: g_{k}=M} e^{\beta \mathbb{1}_{g_{j}=g_{k}}+\delta X_{k}}}{\sum_{k} e^{\beta \mathbb{1}_{g_{j}=g_{k}}+\delta X_{k}}}=\frac{\sum_{k: g_{k}=M} e^{\beta+\delta X_{k}}}{\sum_{k: g_{k}=M} e^{\beta+\delta X_{k}}+\sum_{k: g_{k} \neq M} e^{\delta X_{k}}} \\
& \stackrel{P}{\rightarrow} \frac{M \eta_{M} e^{\beta}}{M \eta_{M} e^{\beta}+(1-M) \eta_{F}}=\frac{M e^{\beta}}{M e^{\beta}+\eta(1-M)}
\end{aligned}
$$

where $\eta_{G}=\mathrm{E}\left[e^{\delta X_{k}} \mid g_{k}=G\right]$ for $G \in\{M, F\}$, and $\eta=\frac{\eta_{F}}{\eta_{M}}$ (so $\eta \gtreqless 1$ when $\mathrm{E}\left[e^{\delta X_{k}} \mid g_{k}=F\right] \gtreqless$ $\mathrm{E}\left[e^{\delta X_{k}} \mid g_{k}=M\right]$. The convergence is by the Law of Large Numbers, assuming characteristics are independent across specialists.

Regardless of gender-biased preferences, if $\eta<1$ male specialists attract a disproportionally high fraction of referrals from both genders (Figure 2). Conversely, if $\eta>1$, female specialists attract more referrals, so whether $P\left(M_{m}\right)$ and $P\left(M_{f}\right)$ are each greater or smaller than $M$ depends on $\eta$. In (2) too, $P\left(M_{m}\right)=P\left(M_{f}\right)$ if and only if preferences are unbiased, i.e., $\beta=0$. So, Proposition 1 also holds for the heterogeneous case.

PROPOSITION A1 (Nonidentificiation of gender-specific gender bias): Consider the model:

$$
\underset{k \in K}{\operatorname{argmax}} U_{j}(k)=\beta_{f} \mathbb{1}_{g_{j}=f, g_{k}=F}+\beta_{m} \mathbb{1}_{g_{j}=m, g_{k}=M}+\delta X_{j k}+\varepsilon_{j k}
$$

where $\beta_{f}, \beta_{m}$ are gender-specific same-gender preferences and $\varepsilon_{j k}$ is as before independently and GEV distributed. Then $\beta_{f}, \beta_{m}$ and let the common tendency to refer to men, $\eta^{\prime}$ (defined as $\eta$ before), are not separately identified.

For example, the case when $\eta=0.2$ and $\beta_{f}=\beta_{m}=0.1$ (namely, male specialists have a 20 percent higher baseline probability of being chosen by both genders. In addition, doctors choose specialists of their own gender with a 10 percent higher probability) is observationally equivalent to $\eta=0.15, \beta_{f}=0.05, \beta_{m}=0.15$ (male specialists have a 15 percent higher baseline, and female doctors are less likely than male doctors to choose same-gender specialists -5 percent versus 15 percent).

Proof of Proposition A1.

PROOF:

Under model (3), the probabilities of referrals to a male, conditional on the doctor gender are:

$$
P\left(M_{m}\right)=\frac{M}{M+e^{-\beta_{m}} \eta^{\prime}(1-M)} \geq \frac{e^{-\beta_{f}} M}{e^{-\beta_{f}} M+\eta^{\prime}(1-M)}=P\left(M_{f}\right) .
$$

Exactly the same probabilities (4) can be obtained by the following reparameterization: $\beta=$ $\frac{1}{2}\left(\beta_{f}+\beta_{m}\right)$ and $\eta=e^{\beta-\beta_{f}} \eta^{\prime}=e^{\beta_{m}-\beta} \eta^{\prime}$. Namely, we can only identify the average of the samegender bias across men and women, not the gender specific bias, as any bias that is common to both genders is observationally equivalent to an unobserved difference between male and female specialists.

Before considering the proof of Proposition 2, some intuition for why sorting generates homophily can be gained by considering the case of no bias.

CLAIM 1 (Sorting-Based Homophily): Assume zero bias. With sorting, referrals exhibit homophily when pooled across all markets:

$$
P\left(M_{m}\right)>M>P\left(M_{f}\right)
$$

for all $\beta \geq 0$. 


\section{PROOF:}

The overall conditional probability is a weighted average of market-specific conditional probabilities (weights are proportional to both market size and the relative share of male doctors in each market). Using Bayes' rule:

$$
\begin{aligned}
P\left(M_{m}\right) & =\sum_{c \in C} P(c \mid m) P(M \mid m, c)=\sum_{c \in C} \mu^{c} \frac{m^{c}}{m} P(M \mid m, c) \\
& \geq \sum_{c \in C} \mu^{c} \frac{m^{c}}{m} M^{c}=\frac{1}{m} E\left[m^{c} M^{c}\right] \\
& >\frac{1}{m} E\left[m^{c}\right] \mathrm{E}\left[M^{c}\right]=M .
\end{aligned}
$$

The first inequality is due to preferences: $P(M \mid m, c) \geq M^{c}$ (equality being the case $\omega=1$ ), and the second is due to segregation. By symmetry, the same proof works for females.

Note that the definition of sorting extends to the more general case where $K_{j}$ is specific to each doctor as: $\operatorname{Cov}\left(m^{j}, M^{K_{j}}\right)>0$, where $m^{j}=\mathbb{1}_{g_{j}=m}$ and $M^{K_{j}}$ is the fraction of males in $K_{j}$. (This definition is indeed more general, as by covariance decomposition, $\operatorname{Cov}\left[m_{j}, M^{j}\right]=\operatorname{Cov}\left[m^{c}, M^{c}\right]$ under separate markets with common $K_{j}=K^{c}$ in each.) For this more general definition of sorting, the proof follows immediately from Proposition 1: with unbiased preferences $P\left(M_{m}\right)=$ $E\left[M^{j} \mid g_{j}=m\right]>M$, by $\operatorname{Cov}\left[m_{j}, M^{j}\right]>0$.

Now, for the proof of Proposition 2.

PROOF:

$$
\begin{aligned}
P\left(M_{m}\right)-M & =\sum_{c \in C} \mu^{c}\left(\frac{m^{c}}{m} P(M \mid m, c)-\frac{m^{c}}{m} M^{c}+\frac{m^{c}}{m} M^{c}-M^{c}\right) \\
& =\sum_{c \in C} \mu^{c}\left(\frac{m^{c}}{m}\left(P(M \mid m, c)-M^{c}\right)+M^{c}\left(\frac{m^{c}}{m}-1\right)\right) \\
& =\mathrm{E}\left[\frac{m^{c}}{m}\left(P(M \mid m, c)-M^{c}\right)\right]+\operatorname{Cov}\left[\frac{m^{c}}{m}, M^{c}\right] .
\end{aligned}
$$

Where $\mu^{c}$ denotes market size.

Note that this proof only uses Bayes' rule to relate aggregate and market-specific referral probabilities and does not rely on a specific parameterization of these probabilities: it only requires relevant moments to exist.

Proposition A2 restates Proposition 2 using relative homophily.

PROPOSITION A2 (Relative Homophily Decomposition): The overall relative homophily decomposes as follows:

$$
P\left(M_{m}\right)-P\left(M_{f}\right)=\mathrm{E}\left[\frac{m^{c}}{m} P(M \mid m, c)-\frac{1-m^{c}}{1-m} P(M \mid f, c)\right]+\frac{1}{m(1-m)} \operatorname{Cov}\left[m^{c}, M^{c}\right]
$$

\section{PROOF:}

Applying the proof of Proposition 2 to females (by symmetry) and substituting $P\left(M_{f}\right)=1-P\left(F_{f}\right)$ yields :

$$
M-P\left(M_{f}\right)=\mathrm{E}\left[\frac{1-m^{c}}{1-m}\left(M^{c}-P(M \mid f, c)\right)\right]+\operatorname{Cov}\left[\frac{m^{c}}{1-m}, M^{c}\right]
$$


Hence

$$
\begin{aligned}
P\left(M_{m}\right)-P\left(M_{f}\right)= & \mathrm{E}\left[\frac{m^{c}}{m}\left(P(M \mid m, c)-M^{c}\right)+\frac{1-m^{c}}{1-m}\left(M^{c}-P(M \mid f, c)\right)\right] \\
& +\frac{1}{m(1-m)} \operatorname{Cov}\left[m^{c}, M^{c}\right]
\end{aligned}
$$

Proof of Proposition 3.

PROOF:

Pick any male specialist $k$. The demand $k$ faces in market $c$ is obtained by aggregating over all doctors in that market (as all variables are market specific, I suppress the superscript $c$ ):

$$
\begin{aligned}
D_{M} & =\sum_{j \in J} p_{j k}=\sum_{j \in J} \frac{e^{\beta s(j, k)}}{\sum_{k^{\prime} \in K} e^{\beta s\left(j, k^{\prime}\right)}} \\
& =\sum_{j \in J, g_{j}=1} \frac{e^{\beta s(j, k)}}{\sum_{k^{\prime} \in K} e^{\beta s\left(j, k^{\prime}\right)}}+\sum_{j \in J, g_{j}=0} \frac{e^{\beta s(j, k)}}{\sum_{k^{\prime} \in K} e^{\beta s\left(j, k^{\prime}\right)}} \\
& =\frac{1}{N}\left(\sum_{j \in J, g_{j}=1} \frac{1}{M+\omega(1-M)}+\sum_{j \in J, g_{j}=0} \frac{\omega}{\omega M+(1-M)}\right) \\
& =\frac{n}{N}\left(\frac{m}{M+\omega(1-M)}+\frac{\omega(1-m)}{\omega M+(1-M)}\right) .
\end{aligned}
$$

Where $n=|J|$ and $N=|K|$. When $\omega=1$ then $D_{M}=\frac{n}{N}$, which is independent of both $M$ and $m$. Suppose $\omega<1$. To see that ii(a) is true, rewrite:

$$
\begin{aligned}
D_{M} & =\frac{n}{N M}\left(m P\left(M_{m}\right)+(1-m) P\left(M_{f}\right)\right) \\
& =\frac{n}{N M}\left(P\left(M_{f}\right)+m\left(P\left(M_{m}\right)-P\left(M_{f}\right)\right)\right)
\end{aligned}
$$

and note that $\partial D_{M} / \partial m>0$ since $P\left(M_{m}\right)-P\left(M_{f}\right)>0$ for every $\beta>0$. To see that ii(b) is true take the derivative of $D_{M}$ with respect to $M$ :

$$
\frac{\partial D_{M}}{\partial M}=\frac{n(1-w)}{N}(\underbrace{\frac{(1-m) w}{(1-M(1-w))^{2}}}_{\text {Complements }}-\underbrace{\frac{m}{(M+w(1-M))^{2}}}_{\text {Substitutes }}) .
$$

The denominators of the terms labeled "Complements" and "Substitutes" are both positive. Therefore, for $m$ near enough zero, Complements dominates and the derivative $\partial D_{M} / \partial M$ is positive, whereas for $m$ near enough one Substitutes dominates and the derivative is negative. For intermediate values of $m$, the sign of the derivative may depend on $M$.

\section{B. Additional Tables and Figure}




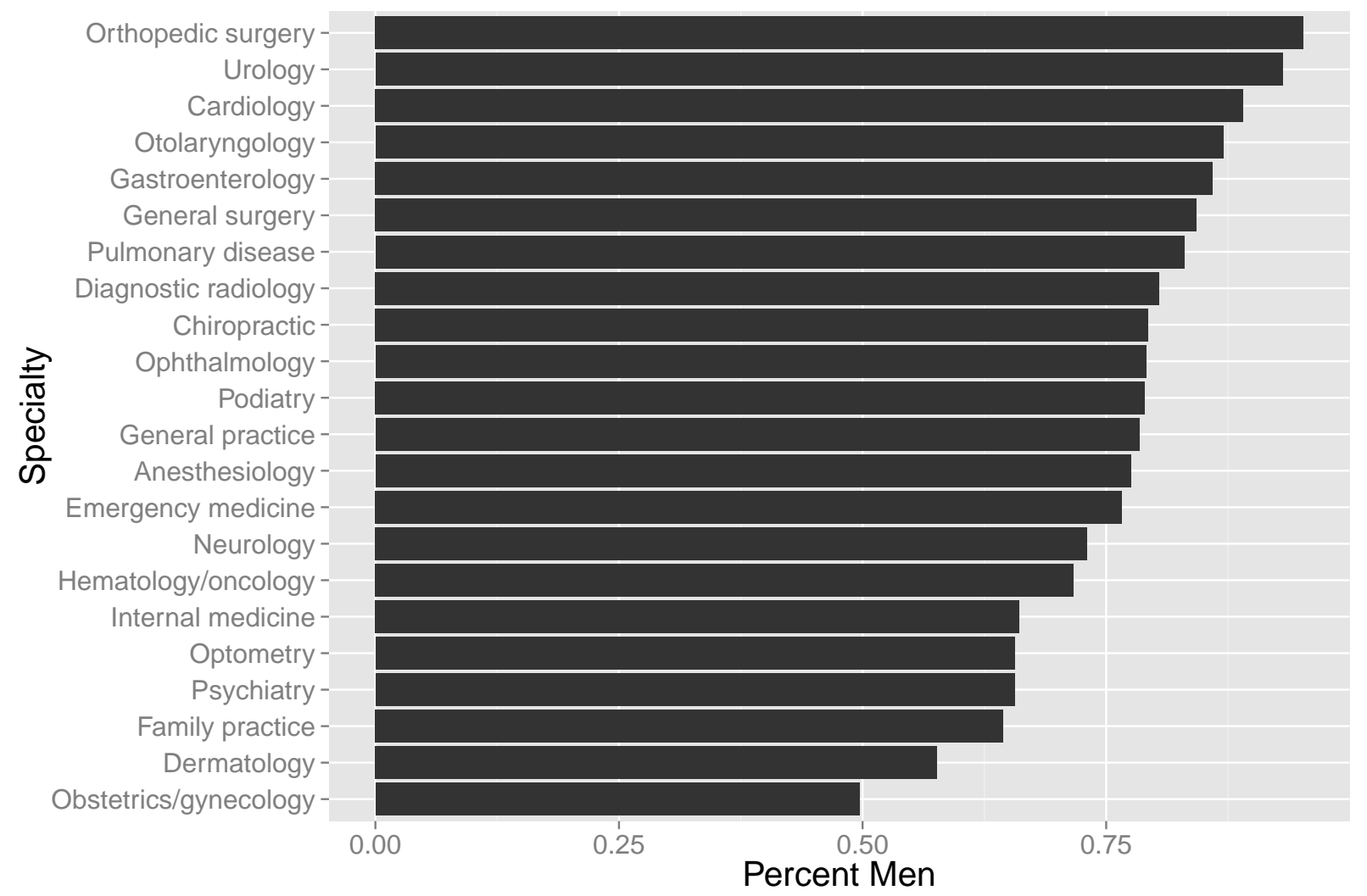

Figure A2. Male Fraction of Physicians in Common Medical Specialties

Notes: Percent of active physicians (with any claims) who are male in 2012, for the most common specialties by overall number of physicians. Columns are sorted so specialties with the greatest male shares are at the top. Source: CMS, author's calculations 


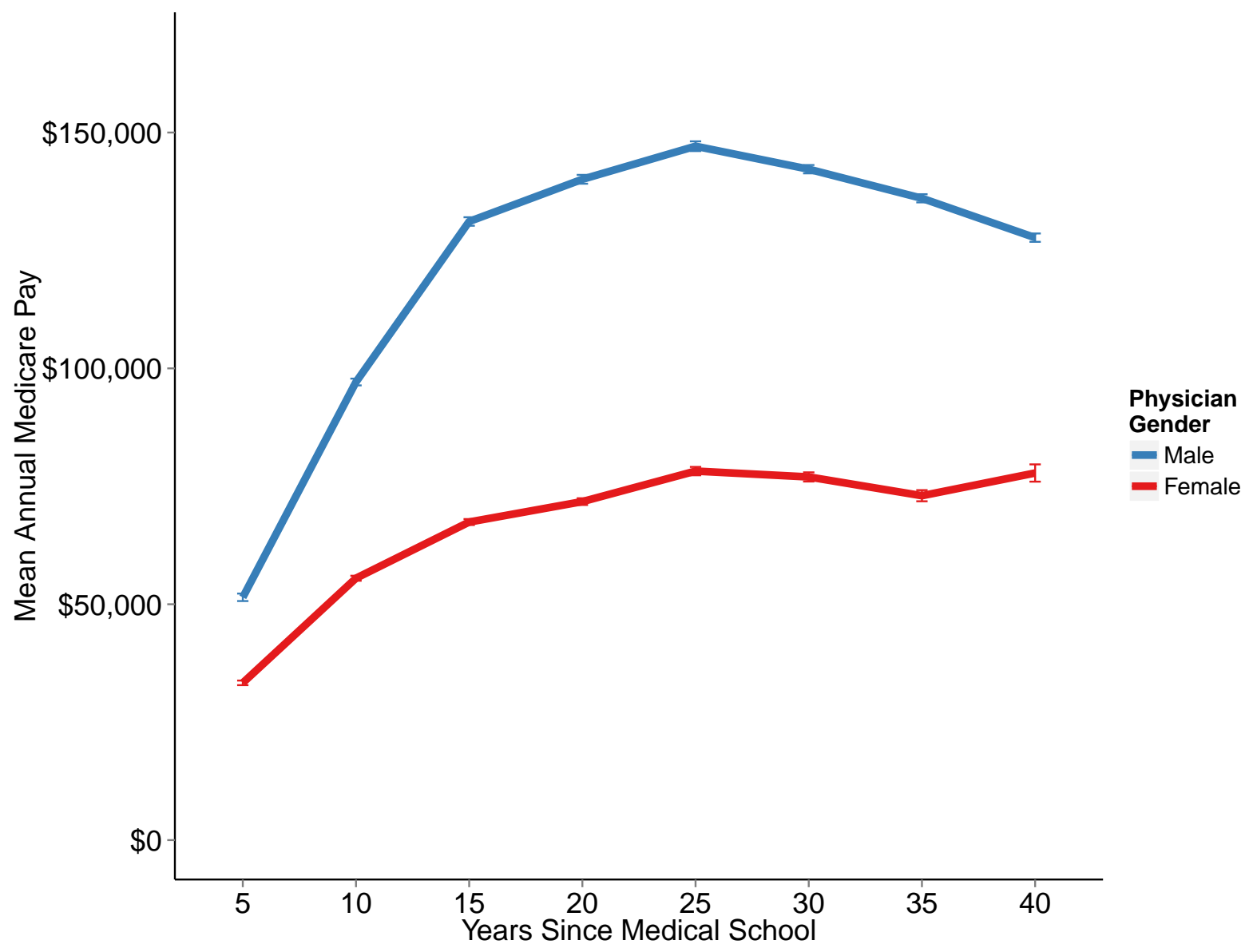

Figure A3. The Unadjusted Gender Pay Gap, by Experience Level

Notes: Source: 20 percent sample of Medicare physician claims for 2012. Mean Annual Medicare Pay is total annual payments (by all payers) to physicians for Medicare services, multiplied by 5 to adjust for sampling. Years are since medical school graduation (bin labels are the range maximum, e.g. 10 stands for 6-10).

Source: CMS, author's calculations 


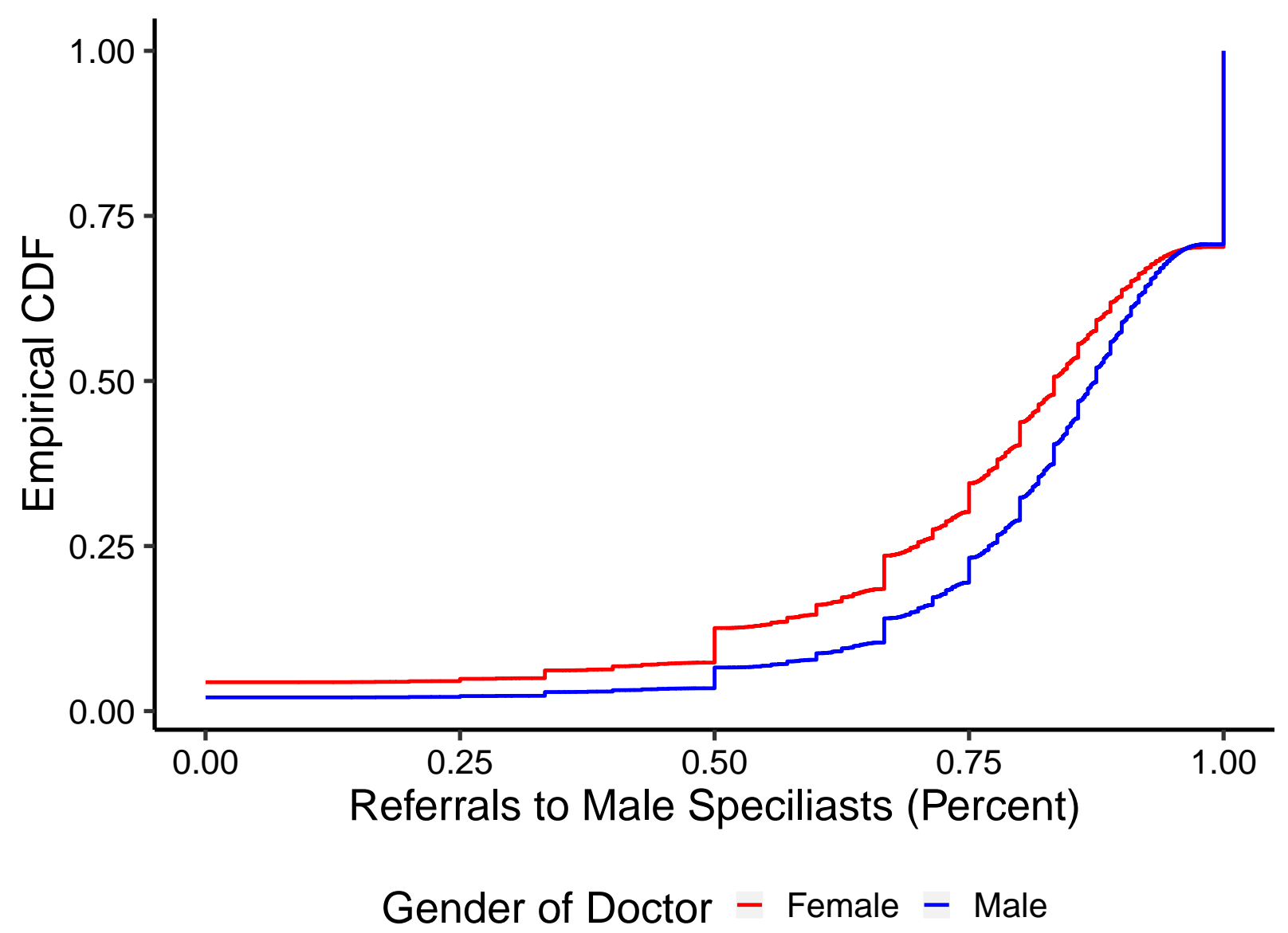

Figure A4. The Distribution of Rates of Referral to Male Specialists

Notes: Empirical cumulative distribution function of $M_{j}$, the fraction of doctor $j$ referrals that are made to male specialists. Source: CMS, author's calculations 


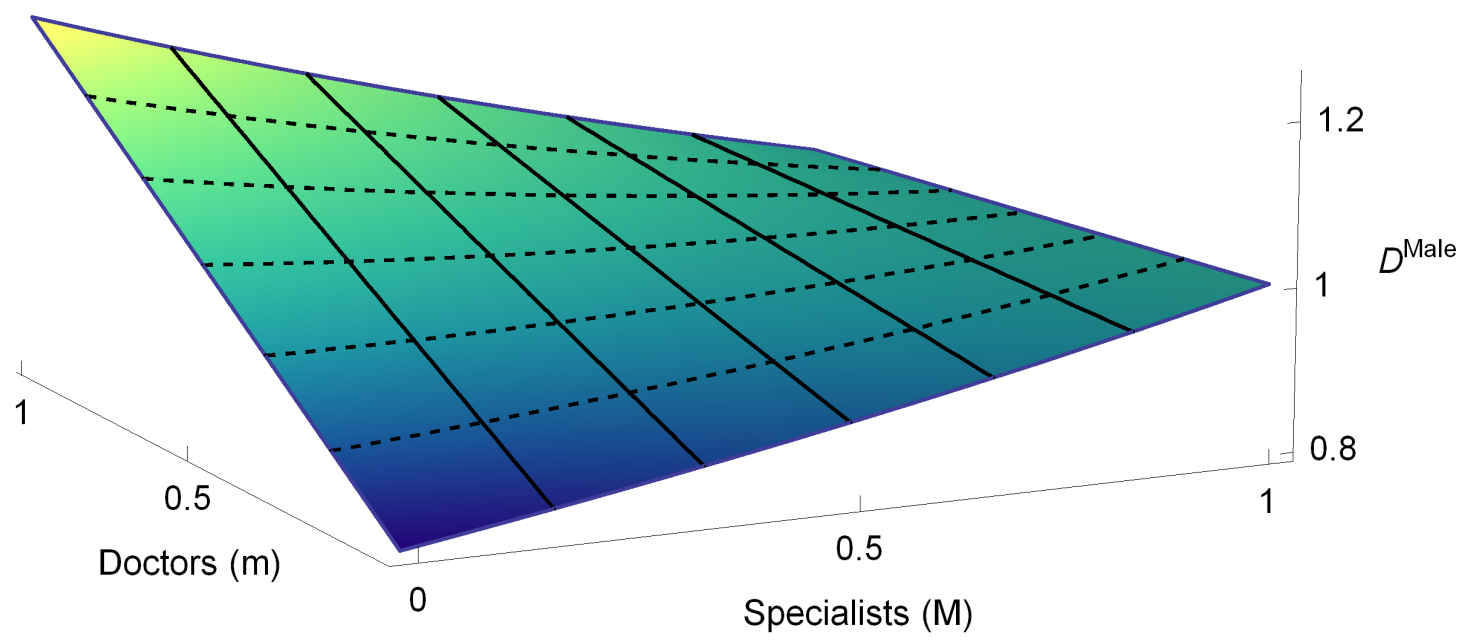

(a) Demand for male specialists over the shares of male doctors and male specialists
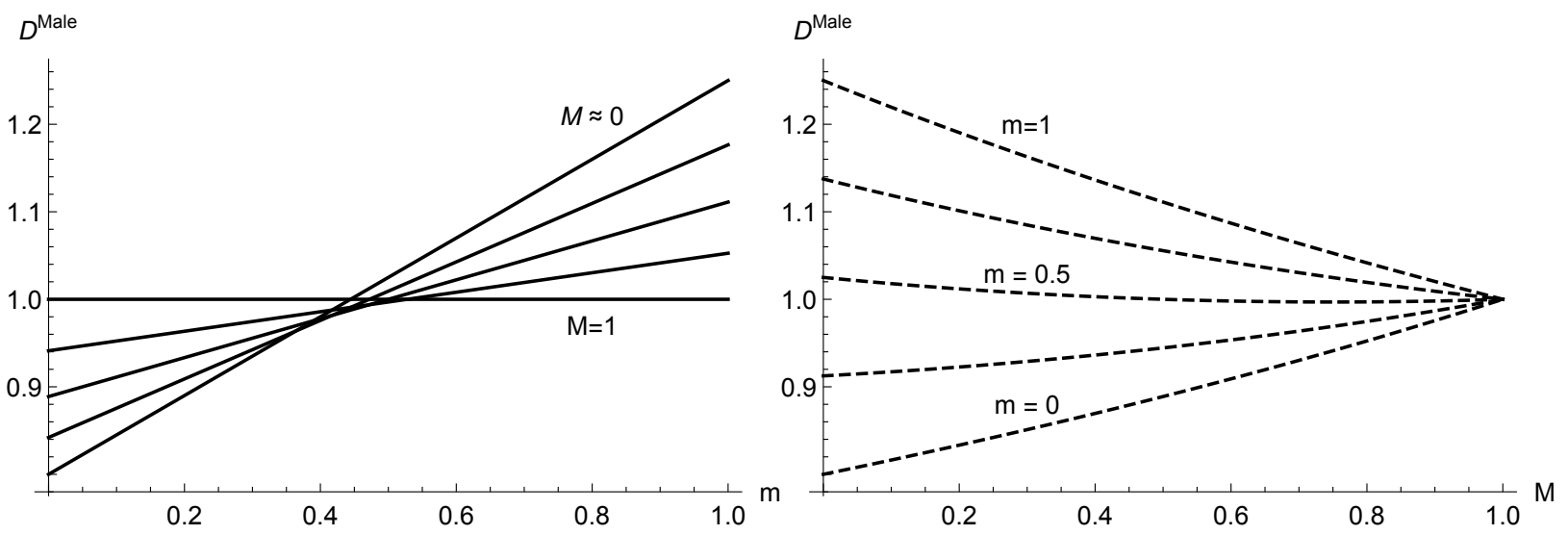

(b) Demand and the share of male doctors, $m$

(c) Demand and the share of male specialists, $M$

Figure A5. Average Specialist Demand with Gender-Biased Preferences

Notes: Average male specialist demand as a function of the fractions of male doctors and male specialists, with gender-biased preferences, i.e. $\beta>0$ (calculated from the model with $\omega=0.8, \eta=1$ ). The surface in Panel (a) depicts the average demand $D^{\mathrm{M}}$, a function of the fractions of both male doctors, $\mathrm{m}$, and male specialists, M. Panel (b) shows different cross sections of $D^{\mathrm{M}}$ for different levels of $M$. Panel (c) shows different cross sections $D^{\mathrm{M}}$ for different levels of $m$. Demand for male specialists is increasing in the share of doctors who are male. Male specialists are complements when only few doctors are men and become stronger substitutes the greater the share of doctors who are are male.

Source: Author's calculations 


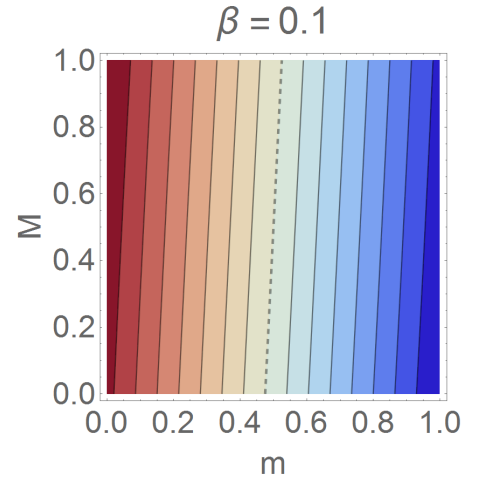

(a)

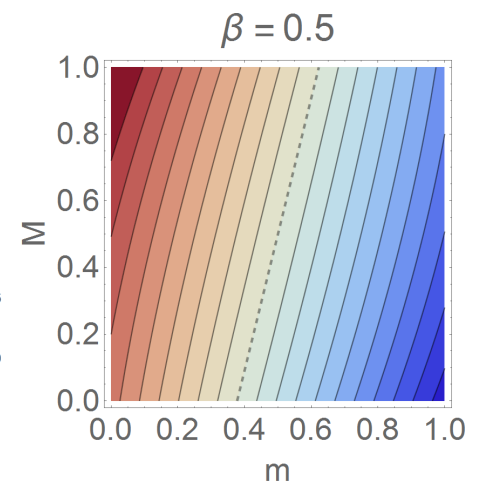

(b)

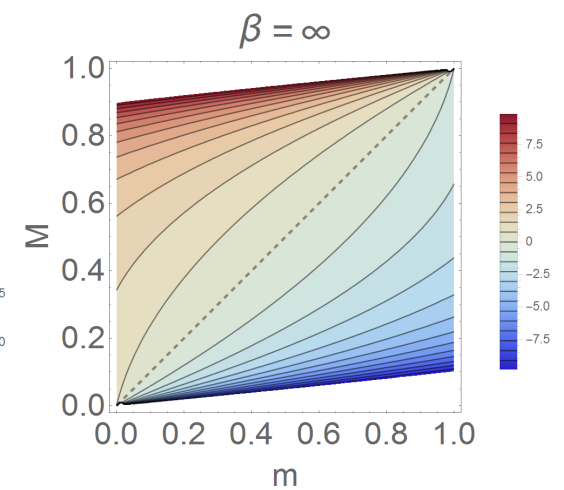

(c)

Figure A6. Counterfactual Gender Earnings Gap with Different Levels of Bias

Notes: Colored contour plots of the gender earnings gap, $D^{F}-D^{M}$, for different fractions of males upstream $m$ and downstream $M$, each for a different level of bias $\beta$. Blue (right) and red (left) darker shades reflect greater demand for male and female specialists, respectively. The zero-gap contours are dashed. For (a) the estimated level of bias for U.S. physicians $(\beta=\hat{\beta}=0.10)$, and even for (b) much higher levels of bias $(\beta=0.50)$, the sign and size of the gender earnings gap mostly depend on the fraction of males upstream. In contrast, for (c) extreme bias $(\beta=\infty)$, a bias that reflects lexicographic preferences, the gap depends on the relative fractions of male doctors and male specialists.

Source: Author's calculations

Table A2-Homophily Estimates with Weighted Links

\begin{tabular}{lcccc}
\hline \hline & \multicolumn{4}{c}{ Dependent variable: } \\
& $\begin{array}{c}\text { Percent } \\
\text { Links }\end{array}$ & Patients & Claims & Dollars \\
\cline { 2 - 5 } & $(1)$ & $(2)$ & $(3)$ & $(4)$ \\
\hline Male Doctor & 0.038 & 0.040 & 0.040 & 0.040 \\
& $(0.001)$ & $(0.001)$ & $(0.001)$ & $(0.001)$ \\
Percent Male Patients & 0.029 & 0.029 & 0.029 & 0.029 \\
& $(0.002)$ & $(0.002)$ & $(0.002)$ & $(0.002)$ \\
Constant & 0.80 & 0.80 & 0.80 & 0.81 \\
Specialty (Doctor) & $(0.003)$ & $(0.003)$ & $(0.003)$ & $(0.003)$ \\
Experience (Doctor) & Yes & Yes & Yes & Yes \\
& & Yes & Yes & Yes \\
\hline Obs. (Doctors) & 384,985 & 384,985 & 384,985 & 383,054 \\
$R^{2}$ & 0.0384 & 0.0394 & 0.0360 & 0.0368
\end{tabular}

Notes: $\quad$ Standard errors in parentheses. OLS estimates of (5) using different definitions of link weights. The first column shows results for unweighted links. Columns 2-4 show results for different weights: number of patients, number of claims, and dollar value of services.

Source: CMS, author's calculations 


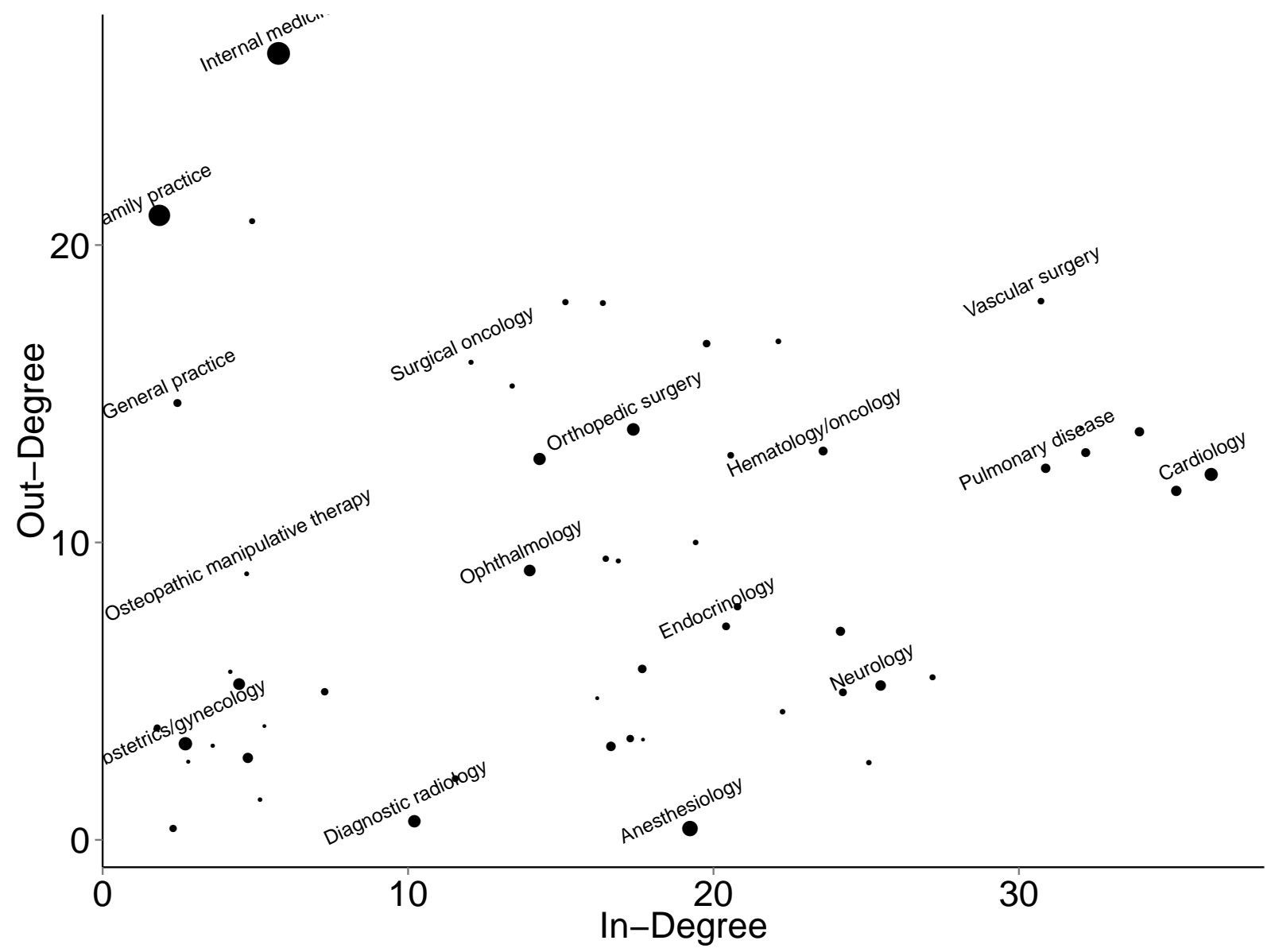

Figure A7. Average Number of Referral Relationships by Medical Specialty

Notes: Degree-heterogeneity is to be expected because doctors in different specialties play different roles in routing patients: some mostly diagnose and refer out, others mostly receive referrals and treat. The figure shows degree distribution by specialty for 2012 referrals: Out-degree is the average number of physicians to whom a physician referred patients during the year. In-degree is the average number of physicians from whom a physician received referrals. Physicians with neither incoming nor outgoing referrals during the year were excluded. Point diameter is proportional to the square root of the number of practitioners in a specialty. Common specialties are labeled. See Table A9 for the data used to generate this figure.

Source: CMS, author's calculations 
Table A3-Estimates of Relative Homophily Using Disaggregated Data

\begin{tabular}{lccccc}
\hline \hline & \multicolumn{5}{c}{$\begin{array}{c}\text { Dependent Variable: } \\
\text { Male Specialist }\end{array}$} \\
\cline { 2 - 6 } & $(1)$ & $(2)$ & $(3)$ & $(4)$ & $(5)$ \\
\hline Male Doctor & $0.045^{* * *}$ & $0.039^{* * *}$ & $0.038^{* * *}$ & $0.035^{* * *}$ & $0.042^{* * *}$ \\
Male Patient & $(0.0008)$ & $(0.0007)$ & $(0.0007)$ & $(0.0007)$ & $(0.0008)$ \\
& & & & $0.021^{* * *}$ & $0.037^{* * *}$ \\
Male Doctor x Male Patient & & & & $(0.0003)$ & $(0.0006)$ \\
& & & & & $-0.019^{* * *}$ \\
Specialty (both) & No & Yes & Yes & Yes & Yes \\
Experience (both) & No & No & Yes & Yes & Yes \\
\hline Obs. (Triples) & $10,545,049$ & $10,545,049$ & $10,127,806$ & $10,127,806$ & $10,127,806$ \\
Clusters (Doctors) & 385,104 & 385,104 & 382,924 & 382,924 & 382,924 \\
R Sqr. & 0.00242 & 0.0689 & 0.0989 & 0.0997 & 0.0998
\end{tabular}

Notes: $\quad$ Standard errors in parentheses. Estimates of relative homophily using one observation for each unique triple of a doctor, a specialist and a referred patient. The sample consists of all such triples for 2012, for a sample of 20 percent of Medicare patients. Standard errors are clustered by doctor.

Source: CMS, author's calculations

Table A4-Residualized Referral Rates to Male Specialists, by Gender of Doctor and Patient

\begin{tabular}{lrrrr}
\hline \hline \multirow{2}{*}{$\begin{array}{l}\text { Mean Residual } \\
\text { Male Specialist) }\end{array}$} & \multicolumn{3}{c}{ Patient Gender } & \\
\cline { 2 - 4 } Doctor Gender & Female & Male & All & Difference (M-F) \\
\hline Female & -0.0705 & 0.0205 & -0.0397 & 0.0910 \\
Male & 0.0329 & 0.0949 & 0.0623 & 0.0620 \\
All & 0.0078 & 0.0835 & 0.0416 & 0.0757 \\
Difference (M-F) & 0.1034 & 0.0744 & 0.1021 & \\
\hline
\end{tabular}

Notes: Average Pearson residuals of logistic regression of a dummy for the specialist being male on doctor and specialist experience and medical specialty. Each cell shows the mean residual among doctors and patients of a given gender. For convenience, the differences between male and female averages are shown in the margins. The sample includes $10,127,806$ patient-doctor-specialist triples in 2012

Source: CMS, author's calculations 


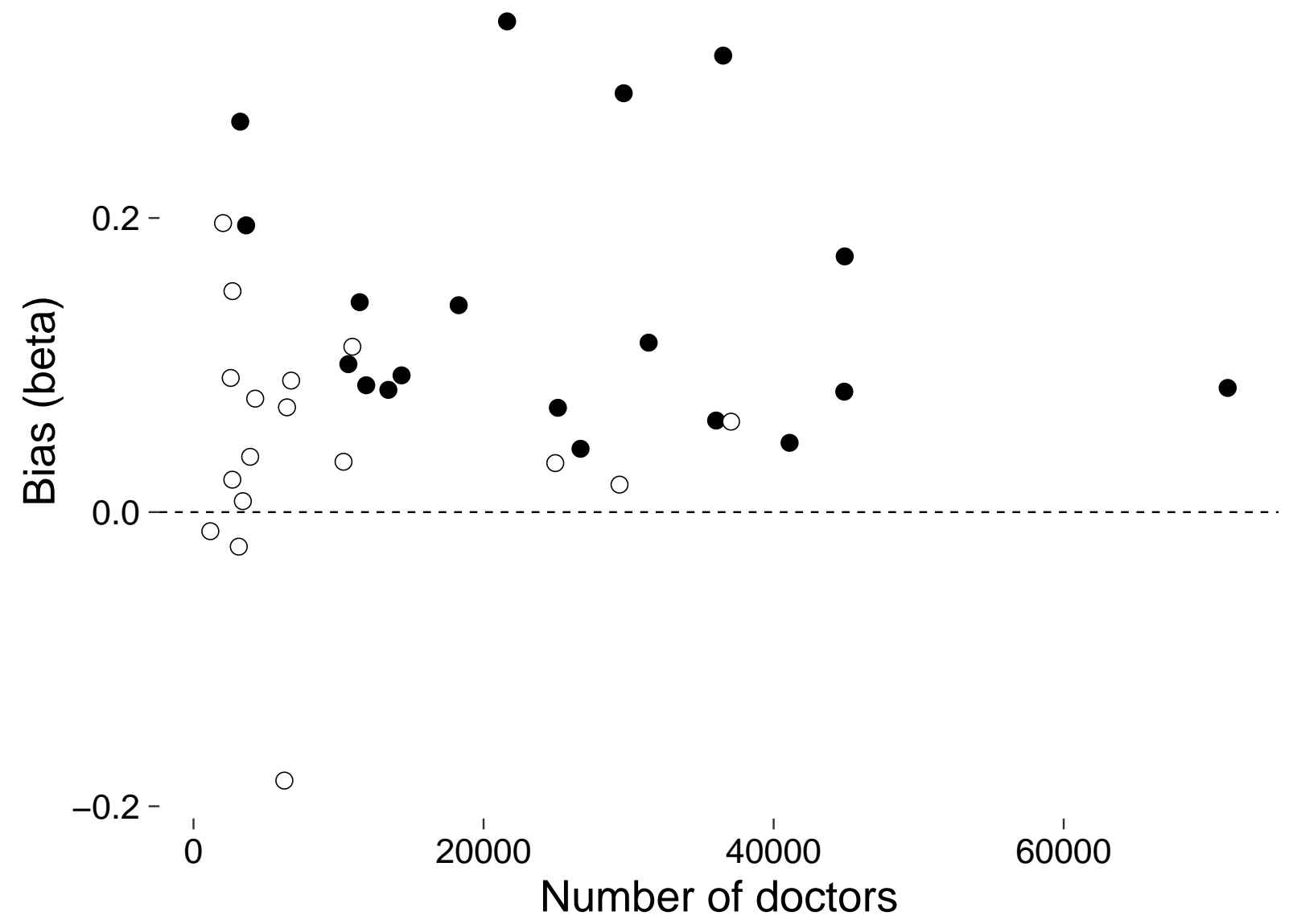

Figure A8. Conditional-Logit Estimates of Gender Bias, by Specialty

Notes: Estimates of $\beta$, the gender bias, from equation (3) with the sample in Table A5, separately for each medical specialty of the receiving physician. Black circles denote estimates that are significantly different from zero $(p<0.05)$. Source: CMS, author's calculations 


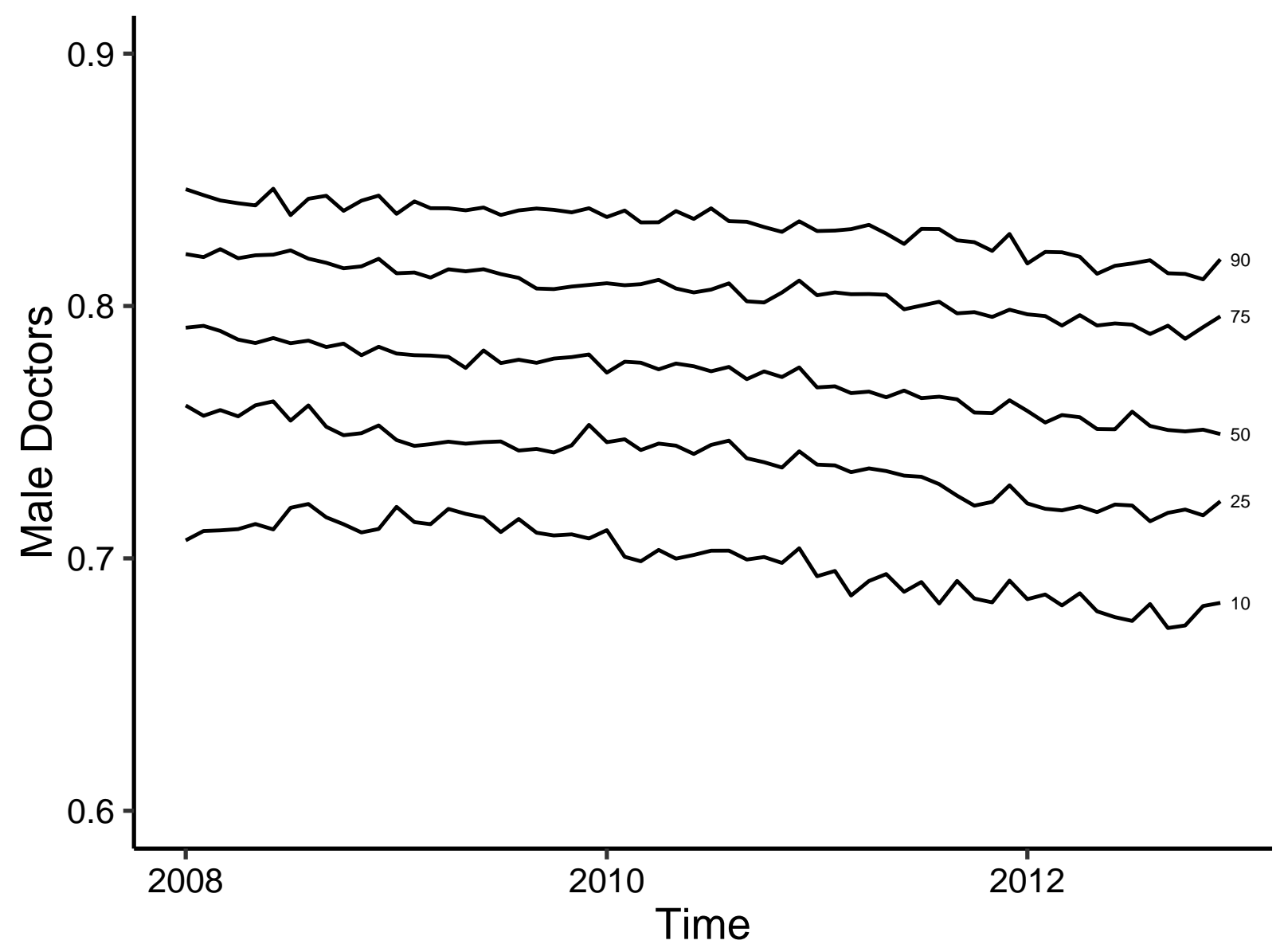

Figure A9. The Fraction of Primary Care Claims Handled by Male Doctors

Notes: Line plots show the 10th, 25th, 50th, 75th and 90th quantiles of the fraction of primary care claims handled by male doctors in each month, calculated across all hospital referral regiosns (HRR). The underlying data are used in the estimation of (13)

Source: CMS, author's calculations 
Table A5-Average Characteristics of Chosen versus Unchosen Specialists

\begin{tabular}{lcc}
\hline \hline & \multicolumn{2}{c}{ Doctor Referred to Specialist } \\
\cline { 2 - 3 } Doctor and Specialist: & Yes & No \\
\hline Same Gender & 0.712 & 0.678 \\
Same Zip Code & 0.280 & 0.0824 \\
Same Hospital & 0.778 & 0.298 \\
Same Group & 0.191 & 0.052 \\
Same Med. School* & 0.107 & 0.0817 \\
Experience Difference (years) & 11.25 & 12.16 \\
\hline Observations (Dyads) & $5,632,166$ & $9,635,750$ \\
& $2,852,950^{*}$ & $4,685,218^{*}$ \\
Clusters (Doctors) & \multicolumn{2}{c}{375,440} \\
& \multicolumn{2}{c}{$242,579^{*}$} \\
\hline
\end{tabular}

Notes: The table describes the sample used for estimating preference bias (as discussed in Sections III.A and IV.B). It shows average characteristics of doctor-specialist pairs. The left, "Yes" column shows data for all specialists chosen by each doctor. For each chosen specialist, the right, "No" column shows data for two randomly sampled specialists not chosen for referrals from the same market (HRR) and medical specialty as the chosen specialist. * denotes the subsample with nonmissing school data. All differences are significant $(p<0.001)$.

Source: CMS, author's calculations 
Table A6-Conditional-Logit Estimates: Referral Probability, with Interaction Terms

\begin{tabular}{|c|c|c|c|c|}
\hline \multirow[b]{2}{*}{ Doctor and Specialist: } & \multicolumn{4}{|c|}{$\begin{array}{c}\text { Dependent variable: } \\
\text { Doctor Referred to Specialist }\end{array}$} \\
\hline & $(1)$ & $(2)$ & $(3)$ & $(4)$ \\
\hline Same Gender & $\begin{array}{c}0.084 \\
(0.002)\end{array}$ & $\begin{array}{c}0.066 \\
(0.004)\end{array}$ & $\begin{array}{c}0.104 \\
(0.004)\end{array}$ & $\begin{array}{c}0.076 \\
(0.006)\end{array}$ \\
\hline Male Specialist & $\begin{array}{c}0.175 \\
(0.002)\end{array}$ & $\begin{array}{c}0.175 \\
(0.002)\end{array}$ & $\begin{array}{c}0.165 \\
(0.004)\end{array}$ & $\begin{array}{c}0.164 \\
(0.004)\end{array}$ \\
\hline Same Hospital & $\begin{array}{c}3.114 \\
(0.004)\end{array}$ & $\begin{array}{c}3.072 \\
(0.005)\end{array}$ & $\begin{array}{c}2.941 \\
(0.005)\end{array}$ & $\begin{array}{c}2.887 \\
(0.007)\end{array}$ \\
\hline Same Hospital x Same Gender & & $\begin{array}{c}0.0598 \\
(0.004)\end{array}$ & & $\begin{array}{c}0.0770 \\
(0.006)\end{array}$ \\
\hline Same Group & $\begin{array}{c}1.346 \\
(0.008)\end{array}$ & $\begin{array}{c}1.372 \\
(0.009)\end{array}$ & $\begin{array}{c}1.320 \\
(0.010)\end{array}$ & $\begin{array}{c}1.344 \\
(0.010)\end{array}$ \\
\hline Same Group x Same Gender & & $\begin{array}{l}-0.039 \\
(0.007)\end{array}$ & & $\begin{array}{l}-0.035 \\
(0.010)\end{array}$ \\
\hline Same Zip Code & $\begin{array}{c}1.074 \\
(0.005)\end{array}$ & $\begin{array}{c}1.065 \\
(0.007)\end{array}$ & $\begin{array}{c}1.054 \\
(0.006)\end{array}$ & $\begin{array}{c}1.047 \\
(0.009)\end{array}$ \\
\hline Same Zip Code x Same Gender & & $\begin{array}{c}0.013 \\
(0.006)\end{array}$ & & $\begin{array}{c}0.010 \\
(0.009)\end{array}$ \\
\hline Similar Experience & $\begin{array}{c}0.128 \\
(0.001)\end{array}$ & $\begin{array}{c}0.120 \\
(0.002)\end{array}$ & $\begin{array}{c}0.131 \\
(0.001)\end{array}$ & $\begin{array}{c}0.123 \\
(0.002)\end{array}$ \\
\hline Similar Experience x Same Gender & & $\begin{array}{c}0.012 \\
(0.002)\end{array}$ & & $\begin{array}{c}0.011 \\
(0.003)\end{array}$ \\
\hline Same Med. School & & & $\begin{array}{c}0.209 \\
(0.004)\end{array}$ & $\begin{array}{c}0.206 \\
(0.007)\end{array}$ \\
\hline Same Med. School x Same Gender & & & & $\begin{array}{c}0.004 \\
(0.008)\end{array}$ \\
\hline Specialist Experience & Yes & Yes & Yes & Yes \\
\hline Obs. (Dyads) & $14,555,821$ & $14,555,821$ & $6,712,241$ & $6,712,241$ \\
\hline Clusters (Doctors) & 367,370 & 367,370 & 242,579 & 242,579 \\
\hline Pseudo R Square & 0.361 & 0.361 & 0.347 & 0.347 \\
\hline
\end{tabular}

Notes: $\quad$ Standard errors in parentheses. Results of conditional logit estimates of (3) for 2012, including interaction terms (denoted by $\times$ ). See Table 5 notes for variable definitions and details.

Source: CMS, author's calculations 
Table A7-Counterfactual Earnings Gaps, Counting as Bias the Common Tendency to Refer to Men

\begin{tabular}{lrrrrrrr}
\hline \hline \multirow{2}{*}{ A. Varying $m$ and $M(\beta=0.1)$} \\
\cline { 2 - 8 } Male Doctors $(m)$ & 0.4 & 0.5 & 0.6 & 0.7 & 0.75 & 0.8 & 0.9 \\
\hline 0.4 & 14.7 & 14.4 & 14.1 & 13.9 & 13.7 & 13.6 & 13.3 \\
0.5 & 16.8 & 16.4 & 16.1 & 15.7 & 15.6 & 15.4 & 15.1 \\
0.6 & 18.9 & 18.4 & 18.0 & 17.6 & 17.4 & 17.2 & 16.8 \\
0.7 & 20.9 & 20.4 & 19.9 & 19.4 & 19.2 & 19.0 & 18.6 \\
0.75 & 21.9 & 21.4 & 20.9 & 20.4 & 20.1 & 19.9 & 19.5 \\
0.8 & 23.0 & 22.4 & 21.8 & 21.3 & 21.1 & 20.8 & 20.3 \\
0.9 & 25.0 & 24.4 & 23.7 & 23.2 & 22.9 & 22.6 & 22.1 \\
\hline
\end{tabular}

B. Varying $\beta$ and $m(M=0.75)$

\begin{tabular}{lrrrrrrr} 
& \multicolumn{7}{c}{ Male Doctors $(m)$} \\
\cline { 2 - 8 } Gender Bias $(\beta)$ & 0.4 & 0.5 & 0.6 & 0.7 & 0.75 & 0.8 & 0.9 \\
\hline-0.05 & 16.7 & 15.7 & 14.8 & 13.9 & 13.5 & 13.0 & 12.1 \\
0 & 15.8 & 15.8 & 15.8 & 15.8 & 15.8 & 15.8 & 15.8 \\
0.05 & 14.8 & 15.7 & 16.7 & 17.6 & 18.0 & 18.5 & 19.4 \\
0.1 & 13.7 & 15.6 & 17.4 & 19.2 & 20.1 & 21.1 & 22.9 \\
0.15 & 12.5 & 15.2 & 18.0 & 20.7 & 22.1 & 23.5 & 26.2 \\
0.2 & 11.1 & 14.8 & 18.5 & 22.1 & 24.0 & 25.8 & 29.4 \\
0.25 & 9.6 & 14.2 & 18.8 & 23.4 & 25.7 & 28.0 & 32.5 \\
\hline
\end{tabular}

C. Varying $\beta$ and $M(m=0.75)$

\begin{tabular}{lrrrrrrr} 
& \multicolumn{7}{c}{ Male Specialists $(M)$} \\
\cline { 2 - 8 } Gender Bias $(\beta)$ & 0.4 & 0.5 & 0.6 & 0.7 & 0.75 & 0.8 & 0.9 \\
\hline-0.05 & 14.2 & 14.0 & 13.8 & 13.6 & 13.5 & 13.4 & 13.2 \\
0 & 16.7 & 16.5 & 16.2 & 15.9 & 15.8 & 15.7 & 15.4 \\
0.05 & 19.3 & 18.9 & 18.6 & 18.2 & 18.0 & 17.9 & 17.5 \\
0.1 & 21.9 & 21.4 & 20.9 & 20.4 & 20.1 & 19.9 & 19.5 \\
0.15 & 24.6 & 23.8 & 23.1 & 22.4 & 22.1 & 21.8 & 21.2 \\
0.2 & 27.2 & 26.2 & 25.3 & 24.4 & 24.0 & 23.5 & 22.8 \\
0.25 & 29.9 & 28.6 & 27.3 & 26.2 & 25.7 & 25.2 & 24.2 \\
\hline
\end{tabular}

Notes: A variant of Table 6 that includes the estimated tendency common to both male and female doctors to refer to male specialists as bias. That is, in addition to same-gender bias, estimated common bias of 0.165 (from the most saturated model in Table 5) is also included, which further shifts demand toward male and away from female specialists.

Source: Author's calculations 
Table A8-Estimates: Link Persistence

\begin{tabular}{|c|c|c|c|c|}
\hline & \multicolumn{4}{|c|}{$\begin{array}{c}\text { Dependent variable: } \\
\text { Any Referrals Next Year }\end{array}$} \\
\hline & \multirow{2}{*}{$\begin{array}{c}\text { Logit } \\
(1)\end{array}$} & \multicolumn{3}{|c|}{ OLS (with Fixed-Effects) } \\
\hline & & $(2)$ & $(3)$ & $(4)$ \\
\hline Same Gender & $\begin{array}{c}0.044 \\
(0.003)\end{array}$ & $\begin{array}{c}0.014 \\
(0.001)\end{array}$ & & \\
\hline Male Doctor & $\begin{array}{c}0.069 \\
(0.004)\end{array}$ & & & \\
\hline Male Specialist & $\begin{array}{c}0.157 \\
(0.003)\end{array}$ & & $\begin{array}{c}0.029 \\
(0.001)\end{array}$ & $\begin{array}{c}0.006 \\
(0.001)\end{array}$ \\
\hline Similar Experience & $\begin{array}{c}0.005 \\
(0.000)\end{array}$ & $\begin{array}{c}0.001 \\
(0.000)\end{array}$ & $\begin{array}{c}0.002 \\
(0.000)\end{array}$ & $\begin{array}{c}0.001 \\
(0.000)\end{array}$ \\
\hline Same Hospital & $\begin{array}{c}0.118 \\
(0.004)\end{array}$ & $\begin{array}{c}0.027 \\
(0.001)\end{array}$ & $\begin{array}{c}0.030 \\
(0.001)\end{array}$ & $\begin{array}{c}0.027 \\
(0.002)\end{array}$ \\
\hline Same Zipcode & $\begin{array}{c}0.159 \\
(0.003)\end{array}$ & $\begin{array}{c}0.097 \\
(0.001)\end{array}$ & $\begin{array}{c}0.092 \\
(0.001)\end{array}$ & $\begin{array}{c}0.076 \\
(0.001)\end{array}$ \\
\hline Same School & $\begin{array}{c}0.088 \\
(0.003)\end{array}$ & $\begin{array}{c}0.013 \\
(0.001)\end{array}$ & $\begin{array}{c}0.015 \\
(0.001)\end{array}$ & $\begin{array}{c}0.014 \\
(0.002)\end{array}$ \\
\hline Constant & $\begin{array}{l}-0.814 \\
(0.004)\end{array}$ & & & \\
\hline Specialty (Specialist) & No & No & Yes & Yes \\
\hline Obs. $(\mathrm{j}, \mathrm{k})$ & $7,255,778$ & $7,204,471$ & $5,734,596$ & 1496658 \\
\hline Rank & 8 & 5 & 58 & 58 \\
\hline$R^{2}$ & & 0.20 & 0.10 & 0.11 \\
\hline N. Cluster & 280,750 & 255,507 & 191,647 & 64,579 \\
\hline FE1 (Doctors) & & 255,507 & 191,647 & 64,579 \\
\hline FE2 (Specialists) & & 237,363 & & \\
\hline
\end{tabular}

Notes: $\quad$ Standard errors in parentheses. Estimates of the persistence of referral relationships using data from $2008-2012$. The sample consists of an observation for each doctor-specialist pair, for the first year a referral is observed in the data. The dependent binary variable is 1 if the doctor also referred to the specialist during the subsequent year. Same gender is a dummy for the specialist and doctor being the same gender. Male specialist/doctor is a dummy for the specialist/doctor being male. Similar Experience is the negative absolute difference in physicians' year of graduation. Column (1) shows estimates of the logit model specified in equation (11). Column (2) shows linear probability model with two-way fixed effects (for doctor and for specialist) in equation (12). Columns (3) and (4) show linear estimates with doctor fixed-effects only, estimated separately for female (3) and male (4) doctors. Sample size is restricted by the availability of medical school data. Results excluding school affiliation are very similar. All standard errors are clustered by doctor.

Source: CMS, author's calculations 
Table A9-2012 Average Degree by Specialty

\begin{tabular}{|c|c|c|c|c|}
\hline & Specialty & In-Degree & Out-Degree & Physicians \\
\hline 1 & Internal medicine & 5.8 & 26.4 & 86,220 \\
\hline 2 & Family practice & 1.9 & 21.0 & 74,638 \\
\hline 3 & Anesthesiology & 19.2 & 0.4 & 33,434 \\
\hline 4 & Obstetrics/gynecology & 2.7 & 3.2 & 22,871 \\
\hline 5 & Cardiology & 36.3 & 12.3 & 21,714 \\
\hline 6 & Orthopedic surgery & 17.4 & 13.8 & 19,411 \\
\hline 7 & Diagnostic radiology & 10.2 & 0.6 & 18,768 \\
\hline 8 & General surgery & 14.3 & 12.8 & 18,011 \\
\hline 9 & Emergency medicine & 4.5 & 5.2 & 16,065 \\
\hline 10 & Ophthalmology & 14.0 & 9.1 & 15,702 \\
\hline 11 & Neurology & 25.5 & 5.2 & 11,469 \\
\hline 12 & Gastroenterology & 35.2 & 11.7 & 11,178 \\
\hline 13 & Psychiatry & 4.8 & 2.7 & 10,861 \\
\hline 14 & Dermatology & 16.6 & 3.1 & 8,624 \\
\hline 15 & Pulmonary disease & 30.9 & 12.5 & 8,272 \\
\hline 16 & Urology & 33.9 & 13.7 & 8,234 \\
\hline 17 & Otolaryngology & 24.2 & 7.0 & 7,666 \\
\hline 18 & Nephrology & 32.2 & 13.0 & 7,105 \\
\hline 19 & Hematology/oncology & 23.6 & 13.1 & 7,019 \\
\hline 20 & Physical medicine and rehabilitation & 17.7 & 5.7 & 6,224 \\
\hline 21 & General practice & 2.5 & 14.7 & 4,853 \\
\hline 22 & Endocrinology & 20.4 & 7.2 & 4,534 \\
\hline 23 & Infectious disease & 24.2 & 5.0 & 4,492 \\
\hline 24 & Neurosurgery & 19.8 & 16.7 & 4,010 \\
\hline 25 & Radiation oncology & 17.3 & 3.4 & 3,933 \\
\hline 26 & Rheumatology & 20.8 & 7.8 & 3,765 \\
\hline 27 & Plastic and reconstructive surgery & 7.3 & 5.0 & 3,759 \\
\hline 28 & Pathology & 2.3 & 0.4 & 3,627 \\
\hline 29 & Allergy/immunology & 11.5 & 2.0 & 2,768 \\
\hline 30 & Pediatric medicine & 1.8 & 3.8 & 2,695 \\
\hline 31 & Medical oncology & 20.6 & 12.9 & 2,507 \\
\hline 32 & Vascular surgery & 30.7 & 18.1 & 2,486 \\
\hline 33 & Critical care & 16.5 & 9.5 & 2,046 \\
\hline 34 & Thoracic surgery & 15.2 & 18.1 & 1,886 \\
\hline 35 & Interventional pain management & 27.2 & 5.5 & 1,655 \\
\hline 36 & Geriatric medicine & 4.9 & 20.8 & 1,597 \\
\hline 37 & Cardiac surgery & 16.4 & 18.0 & 1,526 \\
\hline 38 & Colorectal surgery & 22.1 & 16.8 & 1,161 \\
\hline 39 & Pain Management & 22.3 & 4.3 & 1,055 \\
\hline 40 & Hand surgery & 19.4 & 10.0 & 1,047 \\
\hline
\end{tabular}

Notes: A link represents referral relationships with another physician from any specialty; specialties with less than 1,000 doctors are included but not shown due to space constraints.

Source: CMS, author's calculations 